\title{
Repeat resective surgery in complex pediatric refractory epilepsy: lessons learned
}

\author{
Regina S. Bower, MD, ${ }^{1}$ Elaine C. Wirrell, MD, ${ }^{2}$ Laurence J. Eckel, MD, ${ }^{3}$ Lily C. Wong-Kisiel, MD, ${ }^{2}$ \\ Katherine C. Nickels, MD, ${ }^{2}$ and Nicholas M. Wetjen, MD ${ }^{1}$
}

Departments of ${ }^{1}$ Neurosurgery, ${ }^{2}$ Neurology, and ${ }^{3}$ Radiology, Mayo Clinic College of Medicine, Rochester, Minnesota

\begin{abstract}
OBJECT Resection can sometimes offer the best chance of meaningful seizure reduction in children with medically intractable epilepsy. However, when surgery fails to achieve the desired outcome, reoperation may be an option. The authors sought to investigate outcomes following resective reoperation in pediatric patients with refractory epilepsy, excluding tumoral epilepsies. Differences in preoperative workup between surgeries are analyzed to identify factors influencing outcomes and complications in this complex group.
\end{abstract}

METHODS Medical records were reviewed for all pediatric patients undergoing a repeat resective surgery for refractory epilepsy at the authors' institution between 2005 and 2012. Tumor and vascular etiologies were excluded. Preoperative evaluation and outcomes were analyzed for each surgery and compared.

RESULTS Ten patients met all inclusion criteria. The median age at seizure onset was 4.5 months. Preoperative MRI revealed no lesion in $30 \%$. Nonspecific gliosis and cortical dysplasia were the most common pathologies. The majority of preoperative workups included MRI, video-electroencephalography (EEG), and SISCOM. Intracranial EEG was performed for $60 \%$ for the first presurgical evaluation and $70 \%$ for the second evaluation. The goal of surgery was palliative in 4 patients with widespread cortical dysplasia. The final Engel outcome was Class I in $50 \%$. The rate of favorable outcome (Engel Class I-II) was 70\%. The complication rate for the initial surgery was $10 \%$. However, the rate increased to $50 \%$ with the second surgery, and 3 of these 5 complications were pseudomeningoceles requiring shunt placement ( 2 of the 3 patients underwent hemispherotomy).

CONCLUSIONS Resective reoperation for pediatric refractory epilepsy has a high rate of favorable outcome and should be considered in appropriate candidates, even as a palliative measure. Intracranial EEG monitoring should be considered on initial workup in cases where the results of imaging or EEG studies are ambiguous or conflicting. Epilepsy secondary to cortical dysplasia, especially if the dysplasia is not seen clearly on MRI, can be difficult to cure surgically. Therefore, in these cases, as large a resection as can be safely accomplished should be done, particularly when the goal is palliative. The rate of complications, particularly pseudomeningocele ultimately requiring shunt placement, is much higher following reoperation, and patients should be counseled accordingly.

http://thejns.org/doi/abs/10.3171/2014.12.PEDS14150

KEY WORDS pediatric epilepsy; epilepsy surgery; reoperation; presurgical evaluation; focal cortical dysplasia

$\mathrm{M}$ EDICALLY refractory epilepsy in children can develop from a variety of etiologies and is often challenging to manage. In many cases, resective surgery can offer the best chance of meaningful seizure reduction or elimination. Outcomes depend on a thorough preoperative investigation and careful selection of appropriate surgical candidates. In certain cases, patients who do not have the desired outcome following surgery may undergo an additional workup and are considered candidates for reoperation.

Reported seizure freedom rates after reoperation for epilepsy in adult and mixed-age patient cohorts have ranged widely, from $19 \%$ to $57 \%, 7,10,12,22,24$ The studies also included a wide variety of pathologies, including tumors and vascular lesions. Studies specifically addressing reoperation in pediatric epilepsy patients are sparse.

ABBREVIATIONS EEG = electroencephalography; $\mathrm{EEEG}$ = intracranial EEG; PET = positron emission tomography; SISCOM = subtraction ictal SPECT co-registered to MRI; SOZ = seizure-onset zone; SPECT = single photon-emission computed tomography; $v E E G=$ video-EEG

SUBMITTED March 20, 2014. ACCEPTED December 17, 2014.

INCLUDE WHEN CITING Published online April 24, 2015; DOI: 10.3171/2014.12.PEDS14150.

DISCLOSURE The authors report no conflict of interest concerning the materials or methods used in this study or the findings specified in this paper. 
Some studies in pediatric patients focus on temporal lobe epilepsy, which accounts for only a fraction of pediatric surgical epilepsy cases. However, in these studies seizurefreedom rates following reoperation were reported as $61 \%-100 \% .^{3,17}$ The largest recent study on reoperation for pediatric refractory epilepsy reported a seizure freedom rate of $61 \%$ and a favorable outcome (Engel Class I and II) rate of $83 \%$ among 23 patients. ${ }^{20}$ Other studies have found a rate of reoperation in children of $9.8 \%{ }^{11}$ and seizure freedom following reoperation of $55 \% .{ }^{23}$ However, these studies all included tumoral epilepsies, which tend to be more well-defined and portend a higher likelihood of favorable seizure outcome with complete resection than other epilepsy etiologies. This is true even for patients who have tumoral epilepsy in association with cortical dysplasia; the outcome tends to be better than for those with cortical dysplasia in isolation. ${ }^{5,6,16,19}$

Therefore, we sought to investigate outcomes following resective reoperation in pediatric patients with refractory epilepsy, excluding tumoral epilepsies. In addition, we sought to analyze differences in preoperative workup between surgeries and identify factors influencing outcomes and complications in this complex group.

\section{Methods \\ Patient Selection}

After approval by our institutional review board, a search of our institutional surgical database was conducted to identify pediatric patients who underwent more than 1 resective surgical procedure for the treatment of medically refractory epilepsy, with at least 1 operation occurring between 2005 and 2012. Refractory epilepsy was defined as seizures refractory to at least 3 antiepileptic medications. Patients 18 years of age or younger were included. Resective surgery was defined as focal cortical resection or lobectomy, amygdalohippocampectomy, or hemispherectomy or hemispherotomy. However, patients undergoing hemispherectomy or hemispherotomy as their first procedure were eliminated as it is primarily a disconnection procedure. Patients undergoing corpus callosotomy, multiple subpial transections, vagal nerve stimulator implantation, or deep brain stimulation in addition to their resective surgery were eliminated, as these procedures are considered primarily palliative. Patients with epilepsy secondary to a tumor or tuberous sclerosis were also eliminated.

\section{Preoperative Evaluation}

All patients undergoing resective surgery for intractable epilepsy first underwent a comprehensive evaluation. This included MRI, routine outpatient electroencephalography (EEG), inpatient continuous video-EEG (vEEG) monitoring in most cases, and in some cases single photon-emission computed tomography (SPECT) and/or positron emission tomography (PET), and neuropsychological evaluation. MRI was done with our standard seizure protocol, which evolved over the 7-year period in which these patients were studied. For all patients, high-resolution T1-weighted MR images, such as coronal magnetizationprepared rapid gradient echo (MPRAGE) images, were obtained to provide detailed evaluation of the hippocampal structures and cortices. Additional sequences variably obtained, now standard in the seizure protocol, include a high-resolution sagittal double inversion recovery (DIR), reformatted into coronal and axial planes, an axial diffusion-weighted imaging (DWI) sequence, a coronal fluid attenuated inversion recovery (FLAIR) sequence, an axial fast spin echo T2-weighted sequence, and an axial gradient echo (GRE) sequence. If intravenous contrast material was provided, a post-contrast submillimeter, isotropic 3D T1-weighted sequence was most often obtained. Cortical thickness maps, diffusion tensor imaging (DTI), and PET/MRI are not part of the standard seizure protocol and were not routinely employed. MRI results were determined to be normal or nonlesional when no structural lesions, abnormal signaling, or asymmetry of size or signal in structures could be found. MRI studies demonstrating postoperative changes only, with no other abnormality, were also considered nonlesional. Each patient was discussed at a multidisciplinary epilepsy conference attended by adult and pediatric epileptologists, neurosurgeons, neuroradiologists, and neuropsychologists.

\section{Seizure Outcomes}

Demographic characteristics, seizure etiology, EEG characteristics, surgery performed, Engel outcome classification, postoperative complications, follow-up, and other relevant clinical data were collected by retrospective chart review. Seizure frequencies were recorded by clinicians based on interviews with families at each clinic visit pre- and postoperatively. A standard seizure diary was not used; however, families/caregivers were accustomed to tracking and consistently providing this information.

\section{Statistical Analysis}

Descriptive statistics were used for each variable, including means and standard deviations for continuous variables and frequencies for categorical variables. Summary statistics were calculated using JMP statistical software.

\section{Results \\ Demographics}

Ten patients were identified who met all inclusion criteria. Their demographic and baseline clinical characteristics are presented in Table 1.

\section{Preoperative Workup}

Preoperative MRI was nonlesional, defined as normal MRI, in 3/10 (30\%) (Table 1). Preoperative workup included MRI, SISCOM, and long-term vEEG monitoring in the majority of patients. Two patients also underwent PET scanning. Table 2 shows the frequency with which SISCOM, intracranial EEG (iEEG), and PET were performed, and the rates of concordance with MRI and vEEG monitoring. Concordance was assessed only when both studies were localizing. For example, when SISCOM and MRI were compared, the concordance rate was reported only for those studies in which both were localizing (inde- 
TABLE 1. Patient demographic and baseline clinical characteristics

\begin{tabular}{|c|c|}
\hline Characteristic & Value $^{*}$ \\
\hline \multicolumn{2}{|l|}{ Sex } \\
\hline Male & 4 \\
\hline Female & 6 \\
\hline \multicolumn{2}{|l|}{ Handedness } \\
\hline Right & 7 \\
\hline Left & 2 \\
\hline NA (too young) & 1 \\
\hline \multicolumn{2}{|l|}{ Age at Sz onset (mos) } \\
\hline Median & 4.5 \\
\hline Interquartile range & $1.8-50.3$ \\
\hline \multicolumn{2}{|l|}{ Baseline neurol deficit } \\
\hline None & 7 \\
\hline Hemiplegia & 3 \\
\hline \multicolumn{2}{|l|}{ Baseline cognitive status } \\
\hline Normal & 5 \\
\hline Mild delay & 3 \\
\hline Moderate/severe delay & 2 \\
\hline \multicolumn{2}{|l|}{ Initial MRI findings } \\
\hline Nonlesional & 3 \\
\hline$C D$ & 3 \\
\hline MTS & 1 \\
\hline Encephalomalacia/ischemic changes & 1 \\
\hline Nonspecific T2 abnormality & 2 \\
\hline
\end{tabular}

$\mathrm{CD}=$ cortical dysplasia; $\mathrm{MTS}=$ mesial temporal sclerosis; $\mathrm{NA}=$ not applicable; neurol = neurological; $\mathrm{Sz}=$ seizure.

* Values represent numbers of patients unless otherwise indicated.

terminate vEEG and nonlocalizing studies or findings of postoperative change alone were not included in assessment of concordance). All studies, when localizing, had high rates of agreement with both vEEG and MRI. However, MRI was more often nonlocalizing or nonspecific than vEEG. A detailed analysis of preoperative workup, surgical procedure, pathology, and outcome for each surgery is shown in Table 3.

\section{Surgical Characteristics and Goals}

Surgical characteristics can be seen in Table 4. The most common surgical pathologies were cortical dysplasia $(60 \%)$ and gliosis $(20 \%)$. It was noted that in 4 of the cases the goals for surgery were palliative rather than curative, due to diffuse and widespread ictal onset and interictal spikes in 2 cases, bilateral cortical dysplasia in 1 case, and involvement of the motor strip in 1 case. In these patients, the goal was reduction in the frequency and severity of seizures through resection of the most epileptogenic region.

\section{Outcomes and Complications}

Seizure outcomes and complications following each surgery are shown in Table 4. Engel outcomes and time to seizure recurrence are shown for each patient for each surgery in Table 3. The final Engel outcome was Class I in $50 \%$ of cases overall. The rate of favorable outcome (Engel Class I-II) was 70\% overall, 83\% among the 6 patients with a curative goal, and 50\% among those with a palliative goal. The median duration of follow-up after final surgery was 32.8 months. The complication rate for the initial surgery was $10 \%$. However, the rate increased to $50 \%$ with the second surgery. Following the second surgery, 3 of 5 patients in whom a complication arose developed hydrocephalus resulting in a pseudomeningocele that ultimately required a shunt for treatment. Two of these 3 patients underwent a hemispherotomy. Thus, the complication rate following subsequent surgery was $67 \%$ (2/3) for hemispherotomy patients and 43\% (3/7) for other surgical procedures.

\section{Discussion}

We present here a unique cohort of complex, challenging refractory epilepsy cases involving pediatric patients undergoing reoperation. Preoperative evaluations are examined preceding both surgeries with a focus on identification of factors that may have helped avoid the second operation.

\section{Seizure Outcomes}

Our study demonstrates that seizure outcome following repeat resective operation for pediatric refractory epilepsy is good, with a favorable outcome of Engel Class I or II achieved in a high percentage of cases $(70 \%$ overall, $83 \%$ among those with a curative goal).

Four of the 10 patients undergoing reoperation did so with the clinical expectation of palliation of seizures, rather than cure, due to the seizure-onset zone (SOZ) in-

TABLE 2. Preoperative workup

\begin{tabular}{|c|c|c|c|c|c|c|c|c|}
\hline \multirow[b]{2}{*}{ Study } & \multicolumn{4}{|c|}{$1 s t O p$} & \multicolumn{4}{|c|}{ 2nd Op } \\
\hline & Done $^{*}$ & Localizing† & Concordant w/vEEG $\ddagger$ & Concordant w/ MRI & Done $^{*}$ & Localizing† & Concordant w/vEEG $\ddagger$ & Concordant w/ MRI \\
\hline SISCOM & $8(80)$ & $6(75)$ & $5 / 6(83)$ & $3 / 3(100)$ & $8(80)$ & $6(75)$ & $4 / 5(80)$ & $1 / 1(100)$ \\
\hline PET & $2(20)$ & $2(100)$ & $2 / 2(100)$ & $1 / 1(100)$ & 0 & - & - & - \\
\hline iEEG & $6(60)$ & $6(100)$ & $5 / 6(83)$ & $2 / 2(100)$ & $7(70)$ & $6(86)$ & $6 / 6(100)$ & $2 / 2(100)$ \\
\hline
\end{tabular}

* Number of patients within group (\%) in whom each study was performed.

$\ddagger$ Number of localizing studies (\%) that were concordant with vEEG/MRI, when both that study and vEEG/MRI were localizing (postoperative changes with no other findings, and widespread/bilateral abnormalities on MRI were not considered localizing).

$\dagger$ Numbers of studies done (\%) that were localizing. 


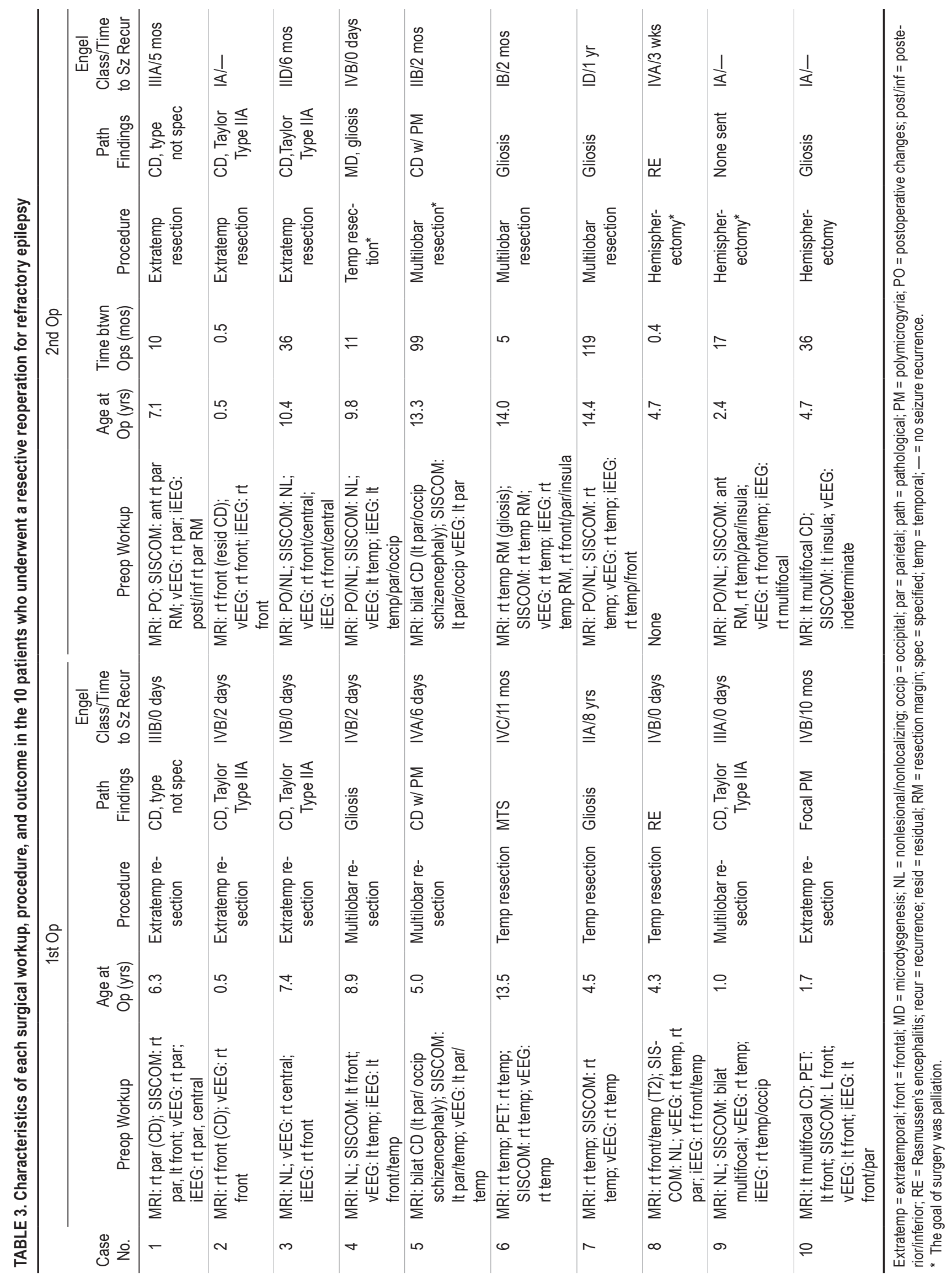


volving eloquent tissue such as the motor strip, diffuse or bilateral onset of seizures, or evidence of widespread malformations of cortical development. In Case 4, iEEG on the initial workup revealed focal onset of the main seizure type, with more diffuse onset of the less common seizure type. Therefore, a focal resection of the SOZ for the main seizure type was undertaken. Subsequent iEEG then revealed diffuse onset and multifocal interictal spikes, with 2 seizure foci, one of which involved eloquent tissue. Therefore, the subsequent resection was palliative, but ultimately did not result in improvement. The patient in Case 5 had bilateral cortical dysplasia seen on initial MRI, although there was only a single focus of seizure onset seen on all studies. Therefore, a focal resection of this area was done, and this resulted in $95 \%$ reduction in seizures for 2 years before the frequency increased again. A subsequent workup suggested a seizure focus at the posterior resection margin but interictal spikes bilaterally, and thus palliation of seizures again was the goal, and focal resection resulted in Engel Class IIB outcome. In this patient's case, iEEG was not done at any time, but it might have been helpful in determining more precisely the SOZ so as to achieve the best outcome possible and avoid a return to surgery. The patient in Case 8 had T2 signal hyperintensity in the mesial temporal lobe consistent with mesial temporal sclerosis, but he also had subtle signal change in the temporal and frontal lobes. His iEEG showed foci in the frontal and temporal lobes, including motor cortex. Because most of his seizures began in the temporal lobe, temporal lobectomy was performed. However, vEEG had shown a seizure focus also in the parietal lobe, with multifocal hemispheric interictal spikes. The pathological findings were was ultimately consistent with Rasmussen's encephalitis, and a hemispherotomy was later done with only Engel Class IVA outcome. Rasmussen's encephalitis had not been suspected initially, but if it had been, perhaps a hemispherotomy could have been considered as a first procedure, thus avoiding reoperation. Unfortunately, in this case, the outcome did not change with hemispherotomy. The patient in Case 9 had a nonlesional MRI, with SISCOM and vEEG pointing to an area that was confirmed by iEEG. A large resection was done encompassing this area, and cortical dysplasia was found on pathological examination. However, only Engel Class IIIA outcome was achieved. Repeat iEEG showed multifocal interictal discharges throughout the entire hemisphere, which had not been seen prior to the first surgery. The MRI findings were still nonlesional, but widespread cortical dysplasia throughout the hemisphere was assumed, so a hemispherectomy was done. This patient might have benefitted from stereotactic EEG initially, to potentially identify multifocal discharges that might have led to performing a hemispherectomy at the outset. Diffusion tensor imaging might have also been helpful in identifying cortical dysplasia on the initial workup.

The goal in these 4 cases was not necessarily cure, but rather significant reduction of seizure frequency and severity by resection of as much as possible of the most epileptogenic area. However, even among these 4 patients, 2 $(50 \%)$ achieved a favorable outcome of Engel Class I or II, underscoring that reoperation should be considered even
TABLE 4. Surgical characteristics, seizure outcome, and complications

\begin{tabular}{|c|c|}
\hline Variable & Value \\
\hline \multicolumn{2}{|l|}{ Age at 1st op (in yrs) } \\
\hline Median & 5.7 \\
\hline IQR & $2.9-10.3$ \\
\hline \multicolumn{2}{|l|}{ Time from Sz onset to op (in yrs) } \\
\hline Median & 3.9 \\
\hline IQR & $1.2-5.4$ \\
\hline AEDs, avg no. preop & 7.5 \\
\hline \multicolumn{2}{|l|}{ VNS } \\
\hline Placed prior to op & 0 \\
\hline Placed after op & 1 \\
\hline No history of VNS & 9 \\
\hline Ketogenic diet preop & 3 \\
\hline \multicolumn{2}{|l|}{ Path findings at 1 st op (\%) } \\
\hline$C D$ & 6 \\
\hline Gliosis & 2 \\
\hline MTS & 1 \\
\hline $\mathrm{RE}$ & 1 \\
\hline None available & 0 \\
\hline \multicolumn{2}{|l|}{ Path findings at 2nd op (\%) } \\
\hline$C D$ & 5 \\
\hline Gliosis & 3 \\
\hline MTS & 0 \\
\hline RE & 1 \\
\hline None available & 1 \\
\hline \multicolumn{2}{|l|}{ Final Engel outcome } \\
\hline Class I & 5 \\
\hline Class II & 2 \\
\hline Class III & 1 \\
\hline Class IV & 2 \\
\hline Favorable outcome (I-II) & $7(70 \%)$ \\
\hline \multicolumn{2}{|l|}{ Complications after 1st op } \\
\hline Infection & 1 \\
\hline Pseudomeningocele requiring shunt & 0 \\
\hline Permanent neurol deficit & 0 \\
\hline Total (\%) & $1(10)$ \\
\hline Complications after 2 nd op & 1 \\
\hline \multicolumn{2}{|l|}{ Infection } \\
\hline Pseudomeningocele requiring shunt & $3^{*}$ \\
\hline Permanent neurol deficit & $1 \dagger$ \\
\hline Total (\%) & $5(50)$ \\
\hline \multicolumn{2}{|l|}{ Follow-up (in mos) } \\
\hline Median & 32.8 \\
\hline IQR & $12.8-64.3$ \\
\hline
\end{tabular}

$\mathrm{AED}=$ antiepileptic drug; IQR = interquartile range; VNS = vagal nerve stimulator.

* Two-thirds were hemispherectomy patients.

$\dagger$ Deficit was unexpected (due to infarct), but the patient recovered with only mild residual deficit. 
among patients with multifocal or bilateral seizure onset in whom reoperation would be considered palliative.

With regard to pathology, we elected to exclude patients with tumors as a cause of their epilepsy, as these lesions tend to be more well-defined and portend better seizure outcomes if they are completely removed and treated, versus more complex epilepsy stemming from such etiologies as cortical dysplasia and encephalitis. 5,6,16,19 Favorable outcome (Engel Class I or II) was achieved in $70 \%$ of patients following reoperation in our study, which is in line with other studies showing favorable outcome in $83 \%$ in children $^{20}$ and $52 \%-81 \%$ in adults. ${ }^{10,21,24}$ However, these studies include patients with tumors as a substantial portion of their cohort. ${ }^{20,23}$ Surgery for tumoral epilepsy tends to have better outcomes than for other epilepsy etiologies. $5,6,10,16,19$ Therefore, it can be more difficult to interpret the data in such studies and apply conclusions to more challenging cases. The majority of patients in our study had pathological findings of cortical dysplasia. However, it is difficult to draw any conclusions regarding outcome based on pathology, as our cohort is small and composed of varied cases, with dysplasia ranging from a small, focal area to widespread, bilateral areas.

\section{Preoperative Evaluations}

Analysis of the preoperative evaluations for both surgeries revealed several interesting points. SISCOM and iEEG were performed frequently at the initial preoperative evaluation, consistent with the frequency of nonlocalizing findings-i.e., nonlesional, encephalomalacia, or nonspecific T2 abnormality-on initial preoperative MRI (60\%). Even in cases in which MRI did reveal cortical dysplasia, when widespread or bilateral, additional studies such as SISCOM or iEEG were required to help identify a specific area of seizure onset. When SISCOM and iEEG were localizing, rates of concordance with localizing vEEG and MRI were high.

The determination of whether and where to perform iEEG monitoring can be somewhat difficult. In our cohort, iEEG was performed at a relatively high rate of $60 \%$ prior to initial surgery. This stands to reason, in that this group tended to consist of complex and challenging cases, in which invasive monitoring is often beneficial when imaging is less helpful. ${ }^{4,8}$ Patients with nonlesional MRI findings or MRI showing widespread encephalomalacia or nonspecific T2 signal abnormalities were the most likely to undergo iEEG monitoring prior to the first operation. Three of the patients who did not undergo iEEG prior to their first surgery did undergo iEEG at the time of their second surgery, and Engel Class I outcome was ultimately achieved in all 3 cases. Of the 6 patients who underwent iEEG at their initial surgery, only 2 did not undergo iEEG at the time of their second surgery, which was a functional hemispherectomy. These findings all suggest that iEEG should be performed at the time of the first surgery if there is any ambiguity in the imaging or inconsistency among studies. Even if preoperative studies are all concordant, but not very precise, iEEG can help to delineate the SOZ with better precision. Although there are risks associated with iEEG, the benefits in carefully selected patients make these risks acceptable. . $^{2,9,18}$
To avoid a return to the operating room to add additional grids, cortical grid coverage should include the entire suspected SOZ as well as large margins. In our cohort, grid coverage was guided by long-term vEEG monitoring, MRI, SISCOM, and/or PET studies. In cases where seizure onset appeared to be diffuse or multifocal, and therefore surgery considered palliative, iEEG was performed to determine whether 1 particular area was dominant and, once resected, would increase the likelihood of seizure improvement.

\section{Complications}

The rate of complications following initial surgery was consistent with other reports in the literature and demonstrates that the group of patients undergoing repeat surgery was not fundamentally different or in some way more prone to complications. However, following their subsequent surgical procedure, the complication rate increased markedly to $50 \%$, with a high incidence of hydrocephalus causing pseudomeningoceles that ultimately required shunt placement. Of the 3 patients in whom this occurred, 2 underwent functional hemispherectomy as their second procedure. This is not an uncommon complication of hemispherectomy, with others reporting rates of $13 \%-39.5 \% .^{1,11,13-15}$ The third patient was an infant at the time of both surgeries ( 6 months old, with just 2 weeks in between surgeries). A higher rate of hydrocephalus as a complication has been shown following craniotomy in infants compared with older children. ${ }^{25}$ The other 2 complications in this group following a second procedure were wound infection and neurological deficit, both of which are unsurprising, given that this was a subsequent procedure, which raises the risk of both.

\section{Limitations}

The main limitation of this study is that the methodology is retrospective. The number of patients, especially those with more than 1 surgery, is small, which makes any associations anecdotal and impacts the ability to draw conclusions applicable on a wider scale. In addition, follow-up was relatively short for several of the patients in our study. We do note, however, that a high proportion of our original patient cohort was maintained in follow-up compared with many other retrospective studies.

\section{Conclusions}

We present a series of cases involving pediatric patients undergoing repeat resective operation following failed surgery for complex refractory epilepsy. After examining each case of reoperation, as well as outcomes and complications, we conclude the following. 1) The rate of favorable outcome following reoperation for pediatric complex refractory epilepsy is good, at $70 \%$ in our series. 2) Reoperation is worthwhile even when deemed palliative due to widespread disease or functional concerns. 3) Intracranial EEG should be considered at the outset for any case in which the findings on preoperative workup are ambiguous or discordant. 4) The complication rate is higher following reoperation, with a high rate of pseudomeningocele formation requiring shunting (30\% in our series). 


\section{References}

1. Basheer SN, Connolly MB, Lautzenhiser A, Sherman EM, Hendson G, Steinbok P: Hemispheric surgery in children with refractory epilepsy: seizure outcome, complications, and adaptive function. Epilepsia 48:133-140, 2007

2. Bauman JA, Feoli E, Romanelli P, Doyle WK, Devinsky O, Weiner HL: Multistage epilepsy surgery: safety, efficacy, and utility of a novel approach in pediatric extratemporal epilepsy. Neurosurgery 56:318-334, 2005

3. Benifla M, Rutka JT, Otsubo H, Lamberti-Pasculli M, Elliott I, Sell E, et al: Long-term seizure and social outcomes following temporal lobe surgery for intractable epilepsy during childhood. Epilepsy Res 82:133-138, 2008

4. Bulacio JC, Jehi L, Wong C, Gonzalez-Martinez J, Kotagal P, Nair D, et al: Long-term seizure outcome after resective surgery in patients evaluated with intracranial electrodes. Epilepsia 53:1722-1730, 2012

5. Cossu M, Fuschillo D, Bramerio M, Galli C, Gozzo F, Pelliccia V, et al: Epilepsy surgery of focal cortical dysplasiaassociated tumors. Epilepsia 54 (Suppl 9):115-122, 2013

6. Cossu M, Lo Russo G, Francione S, Mai R, Nobili L, Sartori I, et al: Epilepsy surgery in children: results and predictors of outcome on seizures. Epilepsia 49:65-72, 2008

7. Cruz VB, Prayson RA: Neuropathology in patients with multiple surgeries for medically intractable epilepsy. Ann Diagn Pathol 16:447-453, 2012

8. Dorward IG, Titus JB, Limbrick DD, Johnston JM, Bertrand ME, Smyth MD: Extratemporal, nonlesional epilepsy in children: postsurgical clinical and neurocognitive outcomes. J Neurosurg Pediatr 7:179-188, 2011

9. Giussani C, Filardi T, Bunyaratavej K, Mai JC, Ogino M, Greene S, et al: Is postoperative CT scanning predictive of subdural electrode placement complications in pediatric epileptic patients? Pediatr Neurosurg 45:345-349, 2009

10. González-Martínez JA, Srikijvilaikul T, Nair D, Bingaman WE: Long-term seizure outcome in reoperation after failure of epilepsy surgery. Neurosurgery 60:873-880, 2007

11. Hemb M, Velasco TR, Parnes MS, Wu JY, Lerner JT, Matsumoto JH, et al: Improved outcomes in pediatric epilepsy surgery: the UCLA experience, 1986-2008. Neurology 74:1768-1775, 2010

12. Holmes MD, Wilensky AJ, Ojemann LM, Ojemann GA: Predicting outcome following reoperation for medically intractable epilepsy. Seizure 8:103-106, 1999

13. Kwan A, Ng WH, Otsubo H, Ochi A, Snead OC III, Tamber MS, et al: Hemispherectomy for the control of intractable epilepsy in childhood: comparison of 2 surgical techniques in a single institution. Neurosurgery 67 (2 Suppl Operative):429-436, 2010

14. Lew SM, Koop JI, Mueller WM, Matthews AE, Mallonee JC: Fifty consecutive hemispherectomies: outcomes, evolution of technique, complications, and lessons learned. Neurosurgery 74:182-194, 2014

15. Lew SM, Matthews AE, Hartman AL, Haranhalli N: Posthemispherectomy hydrocephalus: results of a comprehensive, multiinstitutional review. Epilepsia 54:383-389, 2013
16. Lhatoo SD, Moghimi N, Schuele S: Tumor-related epilepsy and epilepsy surgery. Epilepsia 54 (Suppl 9):1-4, 2013

17. Mittal S, Montes JL, Farmer JP, Rosenblatt B, Dubeau F, Andermann F, et al: Long-term outcome after surgical treatment of temporal lobe epilepsy in children. J Neurosurg 103 (5 Suppl):401-412, 2005

18. Onal C, Otsubo H, Araki T, Chitoku S, Ochi A, Weiss S, et al: Complications of invasive subdural grid monitoring in children with epilepsy. J Neurosurg 98:1017-1026, 2003

19. Palmini A, Paglioli E, Silva VD: Developmental tumors and adjacent cortical dysplasia: single or dual pathology? Epilepsia 54 (Suppl 9):18-24, 2013

20. Ramantani G, Strobl K, Stathi A, Brandt A, Schubert-Bast $\mathrm{S}$, Wiegand $\mathrm{G}$, et al: Reoperation for refractory epilepsy in childhood: a second chance for selected patients. Neurosurgery 73:695-704, 2013

21. Salanova V, Markand O, Worth R: Temporal lobe epilepsy: analysis of failures and the role of reoperation. Acta Neurol Scand 111:126-133, 2005

22. Schwartz TH, Spencer DD: Strategies for reoperation after comprehensive epilepsy surgery. J Neurosurg 95:615-623, 2001

23. Shaver EG, Harvey AS, Morrison G, Prats A, Jayakar P, Dean $\mathrm{P}$, et al: Results and complications after reoperation for failed epilepsy surgery in children. Pediatr Neurosurg 27:194-202, 1997

24. Siegel AM, Cascino GD, Meyer FB, McClelland RL, So EL, Marsh WR, et al: Resective reoperation for failed epilepsy surgery: seizure outcome in 64 patients. Neurology 63:22982302, 2004

25. von Lehe M, Kim HJ, Schramm J, Simon M: A comprehensive analysis of early outcomes and complication rates after 769 craniotomies in pediatric patients. Childs Nerv Syst 29:781-790, 2013

\section{Author Contributions}

Conception and design: Wetjen, Bower, Wirrell. Acquisition of data: Bower, Wong-Kisiel. Analysis and interpretation of data: Bower, Wirrell. Drafting the article: Bower, Eckel. Critically revising the article: all authors. Reviewed submitted version of manuscript: all authors. Statistical analysis: Bower, Wirrell. Administrative/technical/material support: Bower. Study supervision: Wetjen.

\section{Supplemental Information Previous Presentation}

Portions of this work were presented in poster form at the 67th Annual Meeting of the American Epilepsy Society, Washington, DC, December 2013.

\section{Correspondence}

Nicholas Wetjen, Department of Neurosurgery, Mayo Clinic College of Medicine, 200 First St. SW, Rochester, MN 55905. email: wetjen.nicholas@mayo.edu. 